\title{
Simulation procedure of unconfined seepage with an inner seepage face in a heterogeneous field
}

\author{
WU MengXi*, YANG LianZhi \& YU Ting \\ ${ }^{1}$ Institute of Mechanics, Chinese Academy of Sciences, Beijing 100190, China; \\ ${ }^{2}$ Hydrochina Chengdu Engineering Corporation, Chengdu 610072, China
}

Received May 22, 2012; accepted December 14, 2012; published online May 3, 2013

\begin{abstract}
An inner seepage face phenomenon is given and a numerical simulation procedure has been developed. It may appear at the interface of two materials when an unconfined seepage flows from a porous media to a coarser porous media with a higher permeability. Inaccuracy and divergent problems may arise both in a saturated-only and in a variably saturated analysis while an inner seepage face is not simulated with a special procedure. The position of the seepage face is determined during the nonlinear iteration process and the flux of the inner seepage face nodes is transferred to the downstream side nodes. Validity and efficiency of the procedure are illustrated by the simulation of two dimensional steady state seepage examples of heterogeneous zoned dams which is usually used to validate algorithms. An analysis of a three-dimensional earth core rockfill dam is also presented here. The procedure can also be applied to general transient seepage problems.
\end{abstract}

unconfined seepage, inner seepage face, finite element method, free surface

PACS number(s): 02.70.Dh, 47.11.Fg, 47.56.+r

Citation: Wu M X, Yang L Z, Yu T. Simulation procedure of unconfined seepage with an inner seepage face in a heterogeneous field. Sci China-Phys Mech Astron, 2013, 56: 1139-1147, doi: 10.1007/s11433-013-5071-Z

\section{Introduction}

Numerical methods for the analysis of unconfined seepage problems have been a focus of research interest for both engineers and scientists because of the strong non-linearity and importance in underground water resources, hydraulic and hydropower engineering, and other geotechnical engineering since 1960s. The problem with a free surface was first solved numerically by Taylor and Brown [1] and Finn [2] with the adaptive mesh method which involved iteratively modifying a mesh representing only the saturated soil domain until it conformed to the geometry of the saturated flow region. The adaptive mesh methods often lead to divergent calculations [3,4], and difficulties are usually encountered in problems with several types of materials near

*Corresponding author (email: wumx@imech.ac.cn) the free surface [5]. To avoid these difficulties, approaches which only change the meshes intersecting with the free surface while keeping the other meshes unchanged [6-8] were presented. Mesh-fixed approaches were also carried out, e.g., the residual flow scheme presented by Desai [9], a model in which an element intersecting with a free surface is treated to be an element with composite materials proposed by Bathe [10]. These approaches which focus the calculation on the saturated zone require the locating of the free surface by an iterative process. Another group of approaches uses unsaturated theory and the calculation domain involves both the saturated and unsaturated zones [11-15]. The conductivity of the material in an unsaturated zone varies with the degree of the saturation or the minus pore pressure. The problem of the location of the free surface iteration in a saturated-only flow simulation is transformed to the iteration of the conductivity of the materials 
in the field. Durative efforts have been made to improve the convergence, iterative efficiency and accuracy of those approaches, particularly for problems in the heterogeneous field [16-21]. Convergence is often difficult to obtain while the free surface crosses the interface of materials with substantially different hydraulic conductivities [22]. An example to simulate the free surface of a rectangular dam with two materials (as shown in Figure 1(a)) is commonly used to verify algorithms [16-21], however, the locations of the free surface near the interface of the materials among these algorithms are seemingly different. The largest distance of the free surfaces among these algorithms along the interface is up to about $40 \%$ of the water head difference between the upstream and downstream of the model. However, the simulation results of unconfined homogeneous fields are usually identical and agree with experiments, for example, the position of the free surface of a homogeneous rectangular dam is validated both in similar experimental [23] and many similar numerical simulations [5,11,23], as shown in Figure 1(b). An investigation to look inside the seepage phenomenon of unconfined heterogeneous seepage field is made, an inner seepage face is defined and a procedure which can improve both the accuracy and iteration efficiency of the simulation result is provided in this report.

\section{Investigation of the seepage phenomenon in a heterogeneous field}

As noted out by Freeze [11], the zero pressure curve is usually not a streamline in a heterogeneous earth dam, a large proportion of the stream tubes may take unsaturated flow routes for some part of their traverse. Also, a saturated-only analysis which takes the zero pressure curve as a zero flux boundary may cause the determined free surface to be quite different from the actual situation. As shown in Figure 1, the heterogeneous dam in Figure 1(a) is composed with two zones. Zone 1 is the same with the dam in Figure 1(b) in geometry and material. Zone 2 is the same in geometry but is ten times the material conductivity as Zone 1 . The water head in the rectangular area of ABDC in Figure 1(a) is not less than that of in Figure 1(b) because Zone 2 blocks the water flow thus raising the water head in the upstream rectangular area. Thus the free surface AE in Figure 1(a) is not lower than that of in Figure 1(b). This can also be obtained intuitively from the comparison of the similar electrical field in Figures 1(a) and (b) under the similar electric potential boundary. The simulated free surface position in Figure 1(a) which is below curve AE is then not considered accurate. It is obvious that the flux through $\mathrm{CD}$ is not sufficient to maintain a free surface in the right area as high as point $\mathrm{E}$, becasue the conductivity in the right area is greater than that in the left area. Thus there a sharp fall of the free surface in Zone 2 next to the interface EF is seen and the simulated free surface with a higher level in the left part of Zone 2 will correspondingly be not accurate. As EF in Figure 1(b) is a seepage face on which the water pressure equals to the ambient air pressure which is usually set to zero, the water pressure at EF in Figure 1(a) is not less than the ambient air pressure. Thus, the left area of EF in Figure 1(a) is saturated. The top part of the right side of EF is above the free surface. If point $\mathrm{O}$ is the free surface position in Zone 2, line EO is

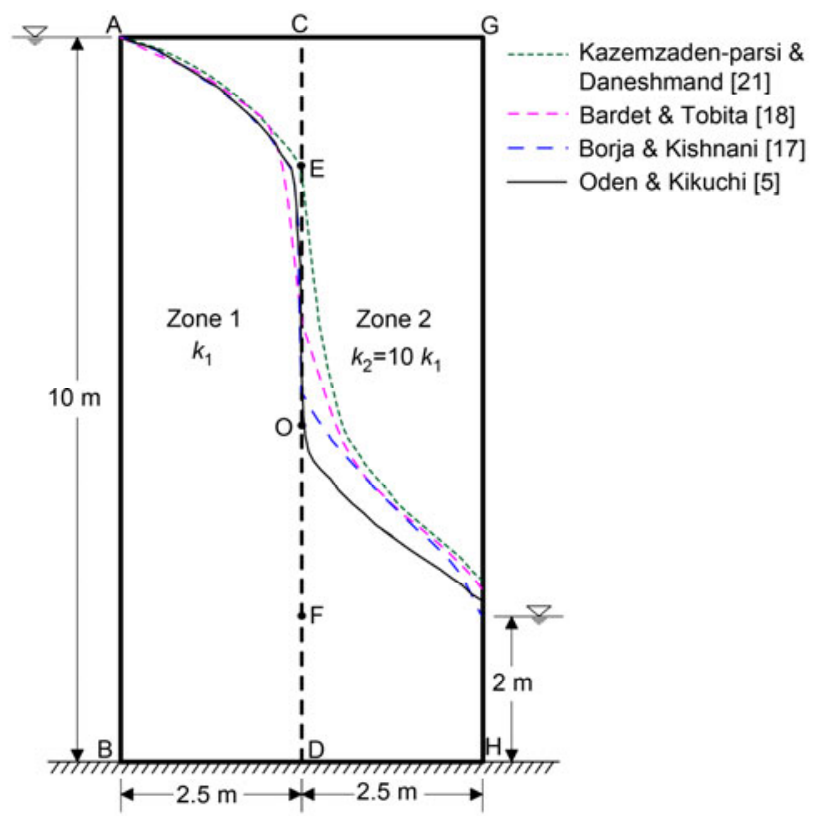

(a)

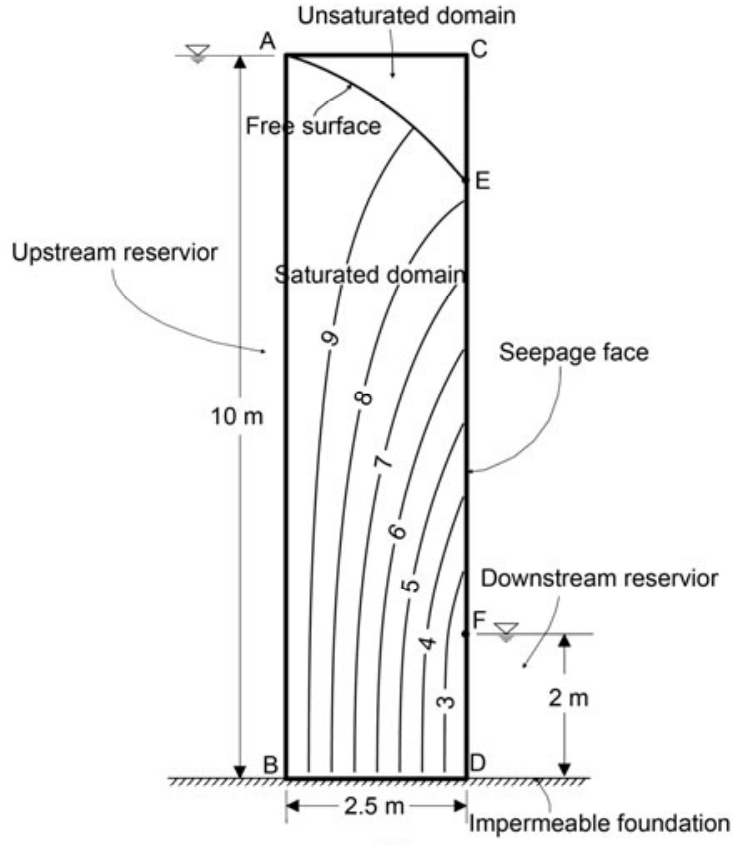

(b)

Figure 1 (Color online) Seepage in (a) a heterogeneous dam and (b) a homogeneous rectangular dam. 
approximately the boundary between saturated and unsaturated zones. The flux flows through line EO can only flow down along the interface within a thin layer as ordinary seepage face in the boundary of the field. For general slope interface situation, there is only a thin layer of saturated soils along EO in the right side of the line in the condition of a vertical or positive slope interface; or there is no saturated soils beneath line EO in the situation of a negative slope interface, because the water flows from the left side either drops down in a discontinuous way or changes to an unsaturated flow in the downside of the line. The seepage character at line EO is similar to a seepage face at the flow boundary. Here EO is a seepage face inside the flow region and is termed an inner seepage face. Special consideration to deal with a seepage face condition is usually necessary, thus giving difficulty in accuracy and convergence in a heterogeneous field without it.

\section{Theory of an interface treatment in an un- confined heterogeneous seepage field}

An boundary of a domain where underground water flow out with the zero water pressure condition (equal to the environmental air pressure which is usually set to zero) is named as a seepage face. The mathematical expression is

$$
\psi=0, q_{n}>0,
$$

where $\psi$ is the pressure head experessed as the heigh of hydrostatic water, $q_{n}$ is the Darcy's flux of a face.

Because the seepage feature at the left side of Line EO in Figure 2 meets the condition of eq. (1), it can be named an inner seepage face which is inside the domain. An inner seepage face is also a boundary between saturated and un-saturated areas like a free surface, with an essential feature difference that there is no flux flow out of a free surface in a saturated-only simulation, however there is flux comes out of an inner seepage face and passed to the next zone. The mathematical expression of a free surface is given as:

$$
\psi=0, q_{n}=0 .
$$

Experimental results show that there is no liquid flow from a porous material with a higher saturation to a coarser material with a larger saturated permeability in an unsaturated area [24]. This principle is widely accepted and has been applied in the design of nuclear waste dumping engineering for decades. Thus, there is no water flows through line CE in Figure 2 but the unsaturated pressure from the left side to the right side alters sharply at a point on line CE.

An inner seepage face may appear while unconfined water flows from one porous media to another with a higher permeability. Both in a saturated-only analysis and in a variably saturated analysis, each node in the seepage face needs two numbers, one for the left area and another for the right area, because the pore pressure at the left side is zero and it is below zero at the right side. The elements located at the two sides of a seepage face are separated in this way and the flux flows out of the left side is equal to the flux infiltrates into the right side. Although the inner seepage face EO in Figure 2 is located at the interface line $\mathrm{CD}$, the range is unknown a priori, changing even with time in transient flow problems, and needing to be determined in the course of iteration. The elements along the material interface which may be within an inner seepage face or above it need to be separated by this way. Because of the difficulty to put two numbers to a node and separate the elements along an interface by this way using a mesh tool, a layer of elements along the interface inside the porous media with the larger conductivity, elements in the rectangle CDD' C' as shown in Figure 2, is used as interface elements to separate the two areas. The range and flux of an inner seepage face need to be determined and the flux needs to be transmitted to the right side of the face.

\section{Procedure for unconfined flow in a heteroge- neous field with an inner seepage face}

Based on the variably saturated seepage method, the procedure for unconfined flow in a heterogeneous field is given as follows.

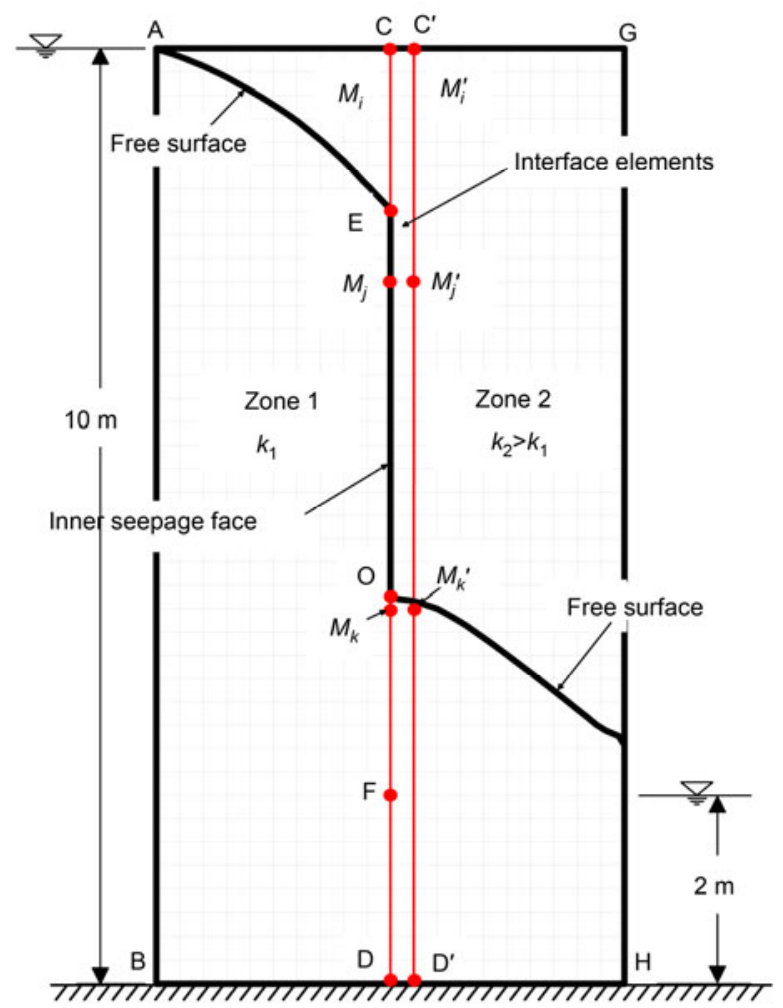

Figure 2 (Color online) Schematic map of interface element and inner seepage face. 


\subsection{Finite element algorithm for variably saturated seepage method}

Water flowing through porous media with a variably saturated seepage method is described by Darcy's equation written in the following form:

$$
q_{i}=-k_{\mathrm{r}}(\psi) K_{i j} \cdot h_{, j}=-k_{\mathrm{r}}(\psi) K_{i j}(\psi+z)_{, j} .
$$

According to the mass-conservative principle and eq. (3), Richards' equation (1931) which governs the variably saturated flow in a porous media can be stated as:

$$
\frac{\partial \theta(\psi)}{\partial t}-\left[k_{\mathrm{r}}(\psi) \cdot K_{i j} \cdot(\psi+z)_{, j}\right]_{, i}=Q .
$$

In eqs. (3) and (4), $q$ is the Darcy flux vector, $k_{\mathrm{r}}(\psi)$ is the relative hydraulic conductivity, $K$ is the tensor of permeability for saturated media, $h=\psi+z$ is the hydraulic head, $\psi$ is the pressure head in dimension of height, $z$ is the elevation above a reference datum, $\theta(\psi)$ is the moisture content and $Q$ is the source/sink term. Subscripts $i, j=1, \cdots, D$ are spatial indices of the Cartesian coordinates, $D$ denotes the number of spatial dimension ( 2 or 3 ) and the summation convention is used for repeated indices.

van Genuchten (1980) [25] equation and Mualem (1976) [26] unsaturated relative permeability function are usually used to describe the relationship of $\theta(\psi)$ and $k_{\mathrm{r}}(\psi)$ with $\psi$.

The finite element algorithm for eq. (4) given by $\mathrm{Wu}$ [15] is:

$$
\left[K_{I J}(\psi)+O(\psi)\right] \psi_{J}^{n}=\tilde{O}(\psi) \psi_{J}^{n-1}+F_{I}(\psi)-K_{I J}(\psi) z_{J},
$$

where

$$
\begin{gathered}
K_{I J}(\psi)=\sum_{e} \int_{\Omega_{e}} k_{\mathrm{r}}\left(\psi^{n, m-1}\right) \cdot K_{i j} N_{J, j} N_{I, i} \mathrm{~d} \Omega, \\
O(\psi)=\sum_{e} \int_{\Omega_{e}} \frac{1}{l} \delta_{i j} \beta\left(\psi^{n, m-1}\right) \frac{\bar{C}\left(\psi^{n, m-1}\right)}{t^{n}-t^{n-1}} \mathrm{~d} \Omega, \\
\tilde{O}(\psi)=\sum_{e} \int_{\Omega_{e}} \frac{1}{l} \delta_{i j} \beta\left(\psi^{n-1}\right) \frac{\bar{C}\left(\psi^{n, m-1}\right)}{t^{n}-t^{n-1}} \mathrm{~d} \Omega, \\
F_{I}(\psi)=\oint_{L}-q_{n} N_{I} d L+\sum_{e} \int_{\Omega} Q N_{I} \mathrm{~d} \Omega .
\end{gathered}
$$

In eqs. (7) and (8):

$$
\begin{gathered}
\beta(\psi)= \begin{cases}1, & \psi \geqslant 0, \\
0, & \psi<0 .\end{cases} \\
\bar{C}\left(\psi^{n}\right)=\left\{\begin{array}{l}
\frac{\theta\left(\psi^{n}\right)-\theta\left(\psi^{n-1}\right)}{\beta\left(\psi^{n}\right) \cdot \psi^{n, m-1}-\beta\left(\psi^{n-1}\right) \cdot \psi^{n-1}} \\
\left|\beta\left(\psi^{n}\right) \cdot \psi^{n}-\beta\left(\psi^{n-1}\right) \cdot \psi^{n-1}\right| \geqslant 0.01, \\
\frac{\theta\left(\psi^{n}\right)-\theta\left(\psi^{n}-0.01\right)}{0.01}, \\
\left|\beta\left(\psi^{n}\right) \cdot \psi^{n}-\beta\left(\psi^{n-1}\right) \cdot \psi^{n-1}\right|<0.01 .
\end{array}\right.
\end{gathered}
$$

In these finite element equations, $l$ is the node number of an element, $\delta_{i j}$ is the Kronecker delta. $N$ is the nodal basis shape function of an element and $I$ or $J$ is the serial number of a node in the element, $n$ is the iterative step of the time $t, m$ is the iterative step for solving the seepage field at the time $t^{n}$.

\subsection{Procedure for Seepage face iteration}

A seepage face is a boundary of the saturated zone with water flow out of the saturated side and the pressure head $\psi$ equals to zero as mathematically expressed in eq. (1). Although the pressure head $\psi$ is known to be zero, the position of a seepage face is unknown a priori, it being a nonlinear boundary condition. An adjustment procedure needs to be used during the iterative solution so that zero pressure is kept inside the seepage face and negative pressures are maintained outside the seepage face, the flux flowing out of the face [12]. During the iteration, the nodes at the boundary (suspended to be a seepage face) with a positive pressure should be altered as Dirichlet nodes with a zero pressure value, the prescribed seepage face nodes with a negative flux need to be identified and treated as nodes outside the seepage face in the next iterative solution of the finite element equations $[15,27]$. The flux of a node can be evaluated by

$$
Q_{I}=-K_{I J} \cdot(\psi+z)_{J},
$$

where $Q_{I}$ is the flux at node $I, z$ is the elevation above a reference datum, $K_{I J}$ is the seepage coefficient tensor assemblage of all the elements [15] shown in eq. (6).

\subsection{Procedure for interface elements}

Special treatment of the interface elements is needed in the numerical iteration process. All interface elements in the area CDD' $\mathrm{C}^{\prime}$ as shown in Figure 2 are treated as ordinary elements as other elements in the field in the first iteration step. In the following steps the seepage feature of an interface element can be identified by the pressure values of its nodes. An interface element in which more than a half nodes with a water pressure no less than zero is treated as an ordinary element which is below or crossing the free surface. These elements need no special treatment. The other elements are on an inner seepage face or in an unsaturated area. These elements do not participate the seepage coefficient tensor assemblage as expressed in eq. (6) at the next iteration step, thus separating the saturated and unsaturated area at the interface in the next iterative solution of the finite element equations expressed in eq. (5). Nodes on the inner seepage face can be determined and its node flux can be obtained according to the procedure mentioned above. As shown in Figure 2, CE is a zero flux boundary, hence node with a negative pressure needs no treatment for no water 
passes it to the downstream area. The EO is an inner seepage face boundary for Zone 1 and the flux boundary for Zone 2. The flux of a node on the inner seepage face needs to be added to the downstream area, e.g., the flux of node $M_{j}$ adds to the opposite node $M_{j}^{\prime}$ therefore the mass conservation law is abided in eq. (5). The flux which flows through the boundary EO is so small that the flow situation from the left side to the right side of the line is similar to the infiltration of dry soil. The relative conductivity at a Gaussian integral point in the element with a small infiltration flux in the two consecutive iteration step changes suddenly during the iteration and the ratio of the conductivity between neighboring Gaussian points at the element may be up to several orders of magnitude. This may cause poor iterative efficiency and serious divergent problems. To avoid this situation the total flux flow out of the seepage face EO can add to a line $M_{k} M_{k}^{\prime}$ which is proximal to the free surface at the downstream side.

All the undetermined boundary conditions including an inner seepage face condition are determined according to the pore water pressure obtained by the last iteration step in the course of the iteration. The procedure to deal with an inner seepage face problem is as follow: (1) An array should be set at first to storage the node numbers of all nodes located at the interface and their attribute values, 1 for nodes below the seepage face which has a positive water pressure, 0 for nodes on the seepage face and -1 for nodes above the seepage face which has a negative pressure. (2) During the iteration, the fluxes of nodes on the seepage face need to be calculated according to eq. (12), the attribute values of those nodes which has a negative flux should be changed from 0 to -1 as a non free surface node. (3) The attribute value of nodes with a positive pressure and -1 value need to be changed to 1 as a free surface node. (4) An interface element which more than a half of the total element nodes are non negative pore pressure nodes are marked as a normal element, non normal elements do not participate the seepage coefficient tensor assemblage at the next iteration step. (5) Node on the interface with 0 attribute value are treated as Dirichlet boundary node with 0 pore pressure and the flux of the node is added to downstream nodes as described in the previous paragraph.

\section{Procedure validation and simulation results comparison with the former analysis}

The performance of the procedure is illustrated by the simulation of three steady seepage examples. The first is the rectangular zoned dam mentioned above, the second a trapezoidal zoned dam, and the third a three dimensional earth core rockfill dam. The results of the analysis are also compared with the former methods in the first and second examples. The procedure can be applied to transient seepage problems for its generality.

\subsection{Example 1: steady seepage field of the rectangular zoned dam}

As shown in Figure 2, 4 nodes square elements with a length of $0.25 \mathrm{~m}$ are used to mesh the domain. It is the same mesh used by Borja et al. [17] and Bardet et al. [18]. The relative permeability of the unsaturated media in Zones 1 and 2 are given in Figure 3. Figure 4(a) shows that the pressure head below the zero pressure line is continuous with a positive pressure, discontinuous at the upper part of the interface of the zones. The position of the zero pressure line differs from that determined in the classic analysis [16-21] as shown in Figure 4(b), and the conflict of the position of zero pressure line with the principle described in section 4 together with Figure 1(b) in the former analysis eliminates. Figure 5 shows that the largest pressure head difference of nodes between two consecutive iteration steps (iterative error) reduces rapidly in the simulation process, indicating a good iteration efficiency of the procedure. If the inner seepage face procedure is not adopted, the iteration is divergent.

If the inner seepage procedure is not adopted by changing the interface elements to ordinary elements, the iterative error with the iterative number and the zero pressure curves in the field calculated in three consecutive iterative steps are shown in Figures 6 and 7 respectively. It shows that the iterative error does not reduce with the iterative number and the convergence is not obtained.

The zero water pressure lines simulated by the method with or without an inner seepage face procedure in the case of coarse mesh are compared in Figure 8. The result simulated with the procedure is coincided with that of in the case of fine mesh as shown in Figure 4(a). Although the simulated result without the procedure is obtained without convergent problem in this coarse mesh case (it is not convergence in the above fine mesh case), the zero pressure line

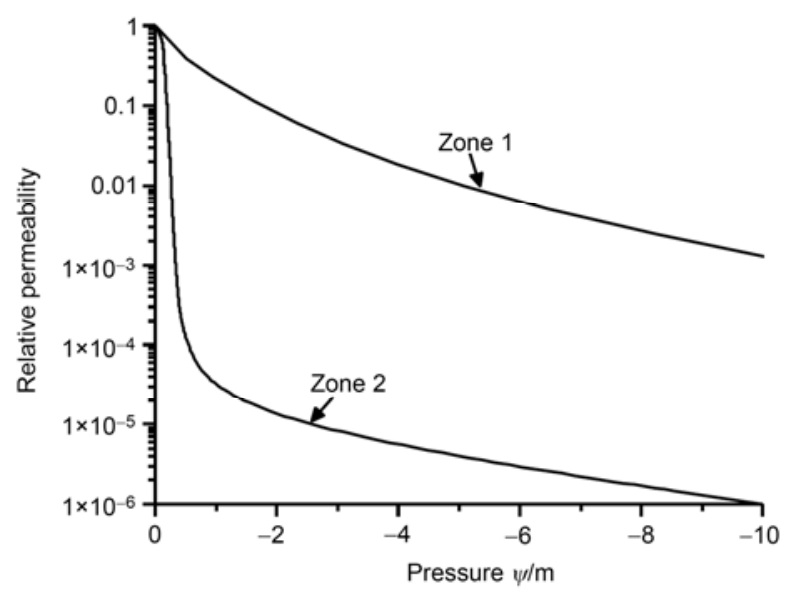

Figure 3 Relative permeability vs. negative pore pressure head (in height of the hydrostatic) of the media in Zones 1 and 2. 


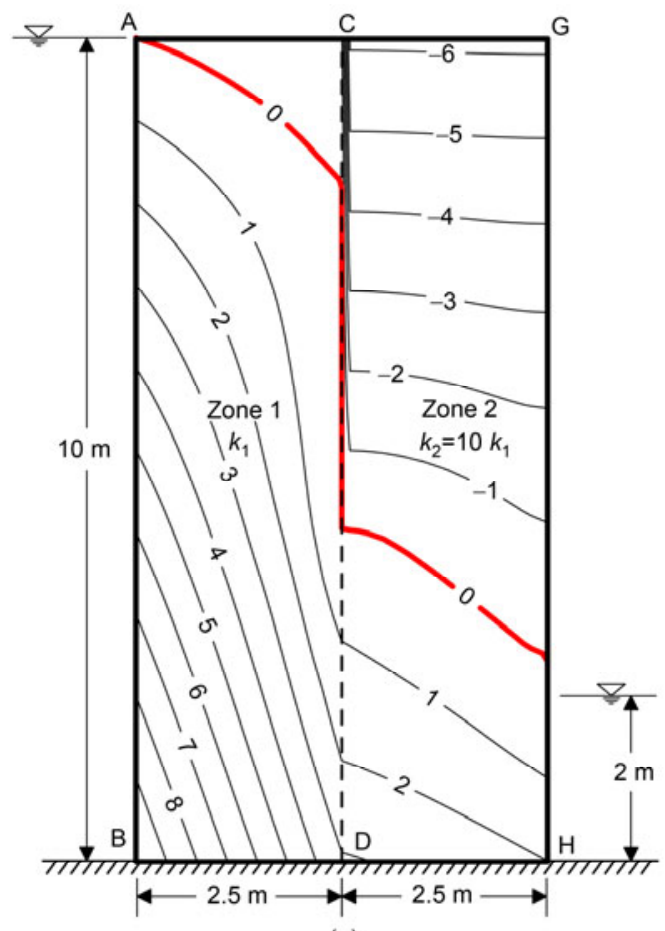

(a)

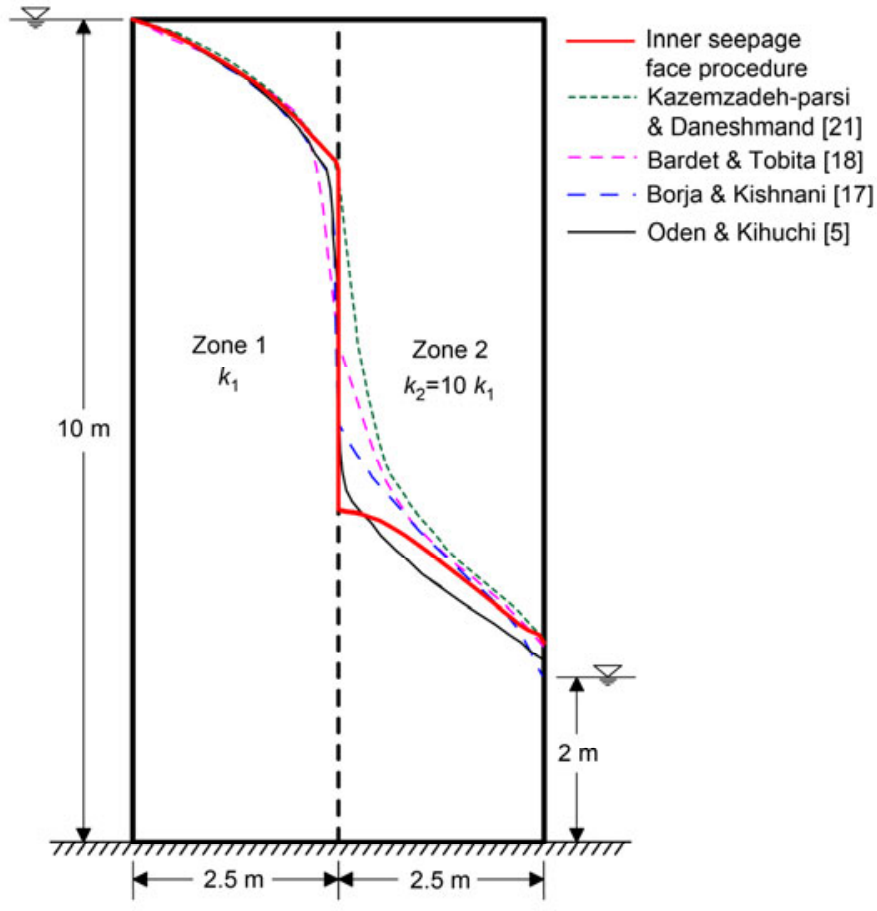

(b)

Figure 4 (Color online) Pressure head contour or zero pressure line in a heterogeneous dam.

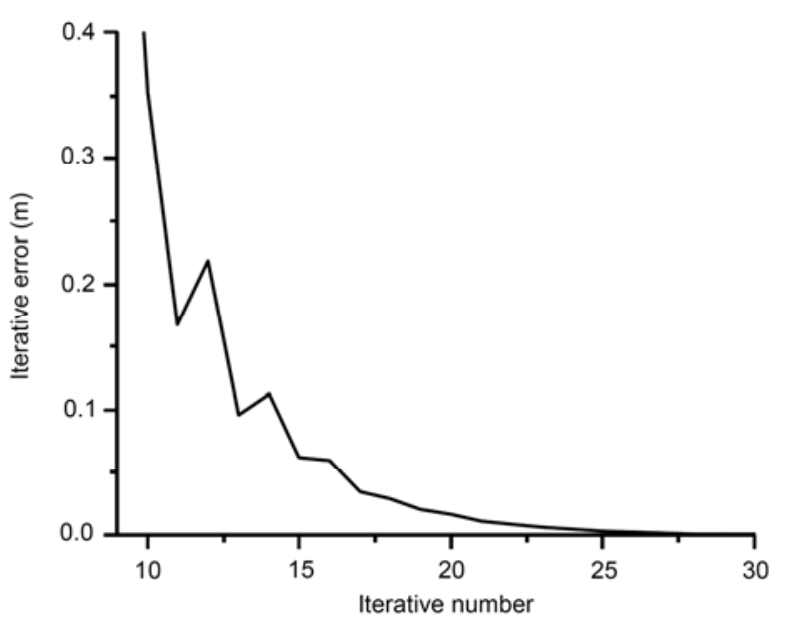

Figure 5 Largest pressure head error between two consecutive iteration steps vs. iterative number.

simulated is obviously not reasonable. Many similar results like the dotted zero pressure line in Figure 8 which does not share a part with the interface of the materials can be found in other reports [16-21].

\subsection{Example 2: steady seepage field of a trapezoidal zoned dam}

Figure 9 shows the zero curve results of a trapezoidal zoned dam. The relative permeability of the unsaturated soil in Zones 1 and 2 is the same with that in Example 1 as given in Figure 3. The zero curves are coincident with that in the saturated-only analysis [21] while the permeability ratio $k_{2} / k_{1}$ equals 1.0 , whereas it differs in the cases of $k_{2} / k_{1}$ is larger than 1.0. The result obtained with an inner seepage procedure is apparently more reasonable than that in the saturated-only analysis [21] for the zero pressure curve is not a streamline in the interface. Reasonable simulation to the inner seepage face is important to the seepage simulation, otherwise not only is the water table position not reasonable, but more importantly, the seepage gradient at the interface of materials which is used to distinguish the internal soil erosion is incorrect.

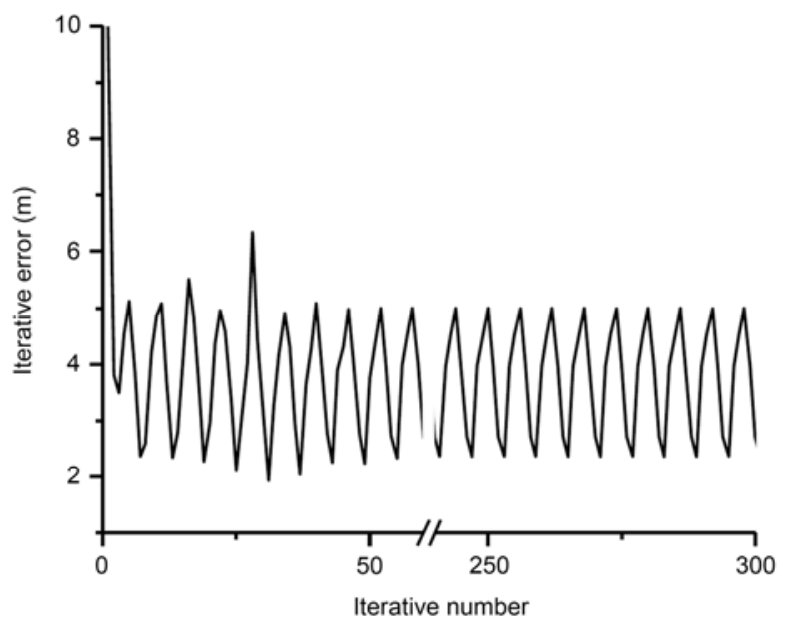

Figure 6 Iterative errors vs. iterative number in the classic analysis. 


\subsection{Example 3: A three dimensional earth core rockfill dam}

Shuang-Jiang-Kou hydropower station is located in Sichuan

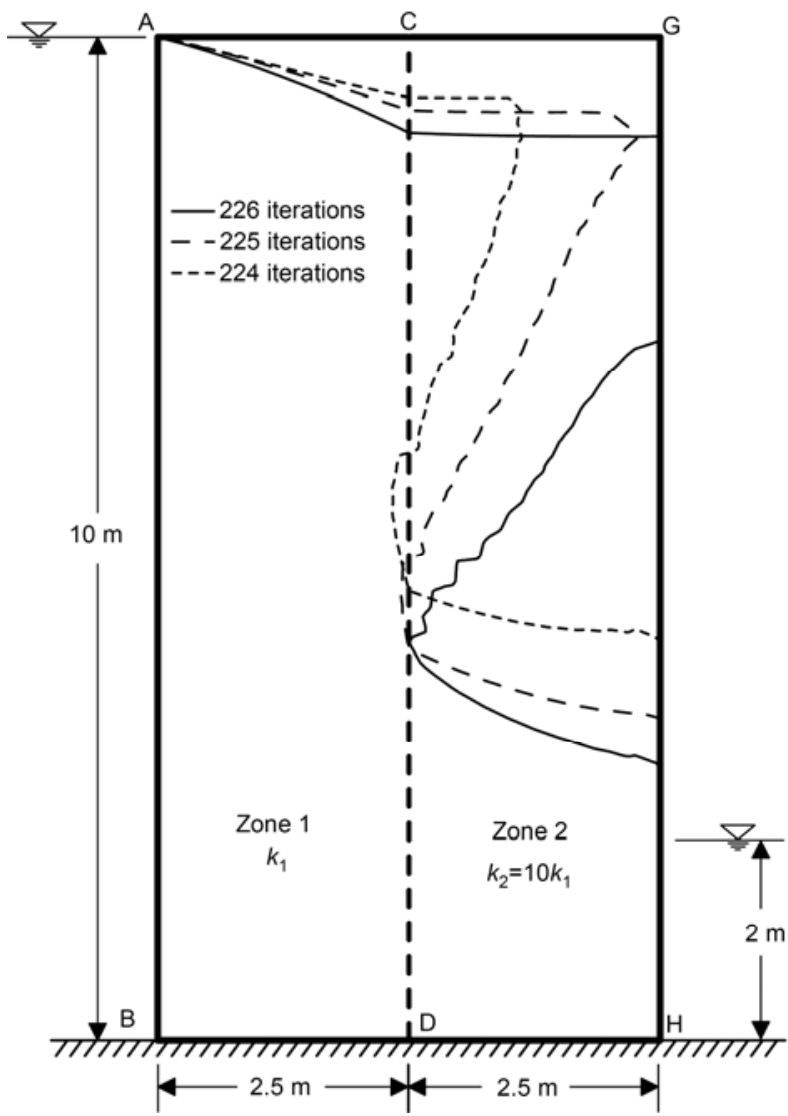

Figure 7 Zero pressure curves in three consecutive iterative steps in analysis without inner seepage procedure.

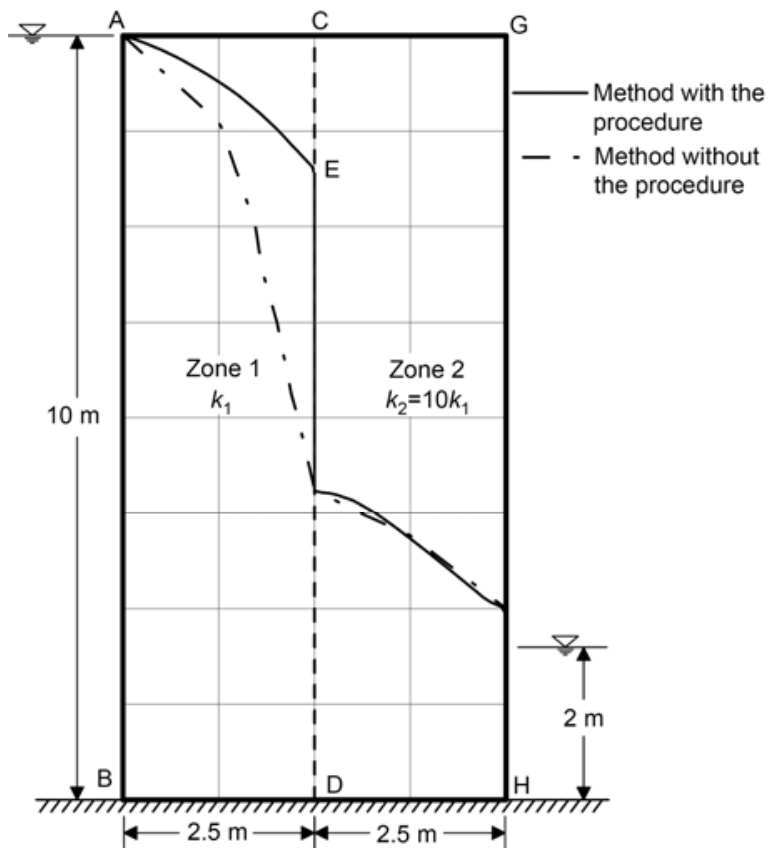

Figure 8 Zero pressure lines by elements with a length $1.25 \mathrm{~m}$ and inner seepage face not adopted.

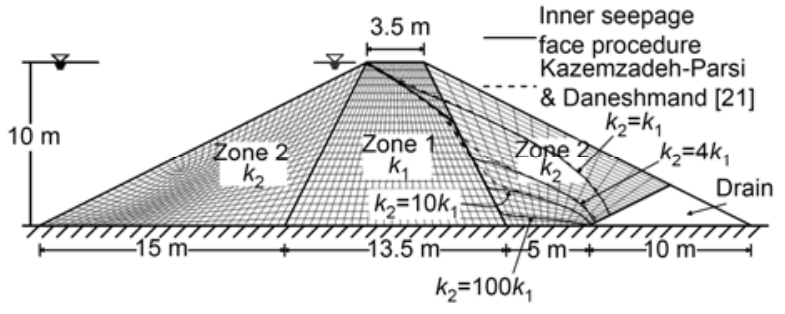

Figure 9 Zero pressure line of the heterogeneous trapezoidal zoned dam.

Province, China, on the upstream of the Dadu River. The power generation workshop is in the underground of the left side bank and the water retaining structure of the reservoir is an earth core rockfill dam with a height of $314 \mathrm{~m}$. A simplified seepage model of the dam is shown in Figure 10, a by-pass seepage elements on the core base is used to simulate the by-pass flux through the bed rock separated by a concrete layer around the core with the same flux going to the downstream riverbed calculated by a large scale model which is $4 \mathrm{~km}^{2}$ in a horizontal plane. The permeability of materials is shown in Table 1, in which the permeability of the by-pass elements is derived from the flux through the base rock. The relative permeability in unsaturated zone involved in this model is given in Figure 11. The pressure contours of the cross sections of the centre valley and of the downstream face of the earth core are shown in Figures 12 and 13, respectively. An inner seepage face appears and occupies a large range of the downstream interface between the core and the filter layer. It can be seen that the inner seepage face procedure is available in three dimensional simulations. Although the ratio of the permeability between the filter and the transition layer and that of between the transition layer and the rockfill layer are also large than 1.0, there is no seepage face appear at their interfaces. Therefore an inner seepage face does not always appear while water flows from a porous material to a coarser porous material.

\section{Conclusion}

An inner seepage face may appear at materials interface in an unconfined heterogeneous seepage field where saturated water flow out of a porous media and goes to a coarser porous media with a higher saturated permeability. A procedure to deal with an inner seepage face is presented. Interface elements and seepage face procedure are used to handle the materials interface. If a special procedure is not adopted to deal with the inner seepage face in this situation, divergent problems will arise usually in a fine mesh, or the simulated results will be inaccurate in a course mesh both in a saturated-only analysis and in a variably saturated seepage analysis. Good iterative efficiency and more reasonable results simulated in the 2-D zoned dams are obtained by the procedure compared with simulations in other reports. The 
Table 1 Permeability of materials in the earth core rockfill dam

\begin{tabular}{cccccccc}
\hline Material & Core & Filter layer & Transition layer & Rockfill & Covering layer & By-pass seepage elements & Concrete \\
\hline Permeability $(\mathrm{m} / \mathrm{s})$ & $7.0 \times 10^{-8}$ & $1.8 \times 10^{-5}$ & $8.0 \times 10^{-4}$ & $1.0 \times 10^{-2}$ & $1.0 \times 10^{-4}$ & $1.1 \times 10^{-4}$ & $1.0 \times 10^{-11}$ \\
\hline
\end{tabular}

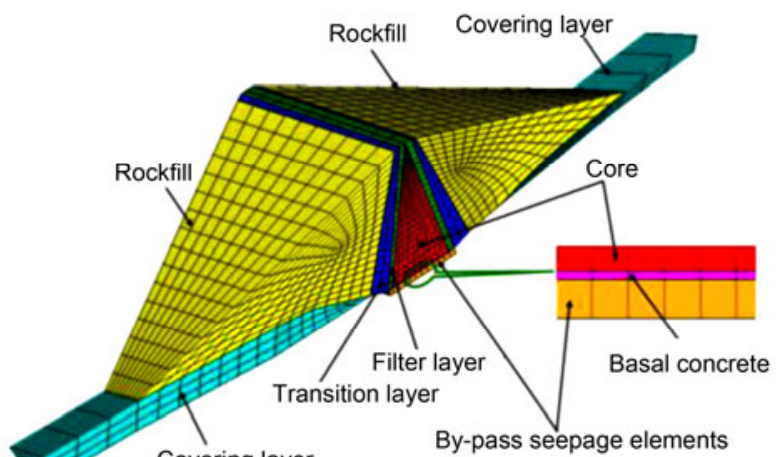

Figure 10 (Color online) Meshes of the earth core rockfill dam.

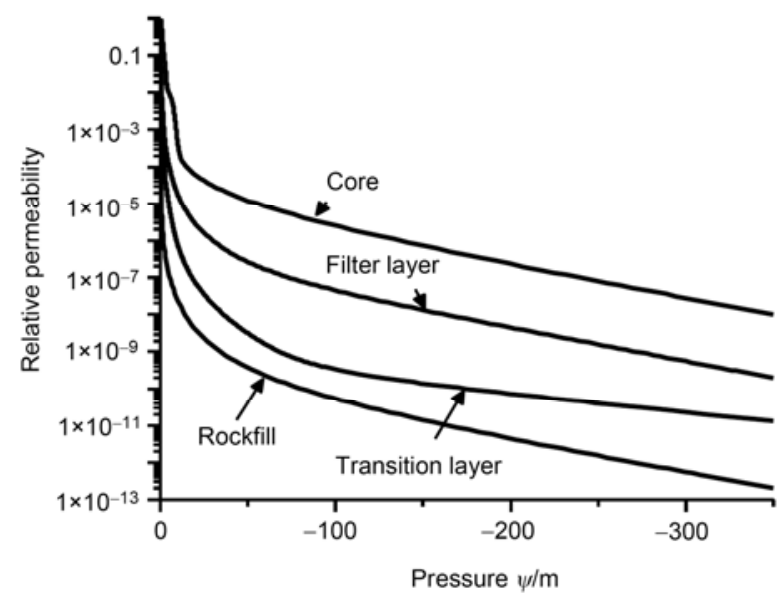

Figure 11 Relationship between relative permeability and negative pore pressure head.

simulation of the 3-D dam example shows the effectiveness of the procedure.

This work was supported by the National Natural Science Foundation of China (Grant No. 10932012), the China-Europe Science and Technology Cooperation Program (Grant No. 0820), and European Commission (Grant No. FP7-NMP-2007-LARGE-1).

1 Taylor R L, Brown C B. Darcy flow solutions with a free surface. J Hydr Div Proc ASCE, 1967, 93(HY2): 25-33

2 Finn W D L. Finite-element analysis of seepage through dams. J Soil Mech Found Div Proc ASCE, 1967, 93(SM6): 41-48

3 Neuman S P, Witherspoon P A. Finite element method of analyzing steady seepage with a free surface. Water Resour Res, 1970, 6(3): 889-897

4 Desai C S. Seepage analysis of earth banks under drawdown. J Soil Mech Found Div Proc ASCE, 1972, 98(SM11): 1143-1162

5 Oden J T, Kikuchi N. Theory of variational inequalities with applications to problems of flow through porous media. Int J Eng Sci, 1980,

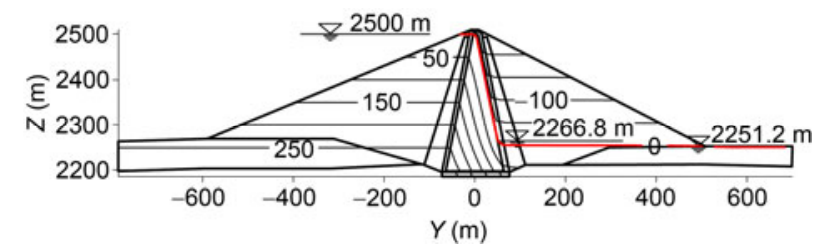

Figure 12 (Color online) Pressure contour in the cross section of the centre valley.

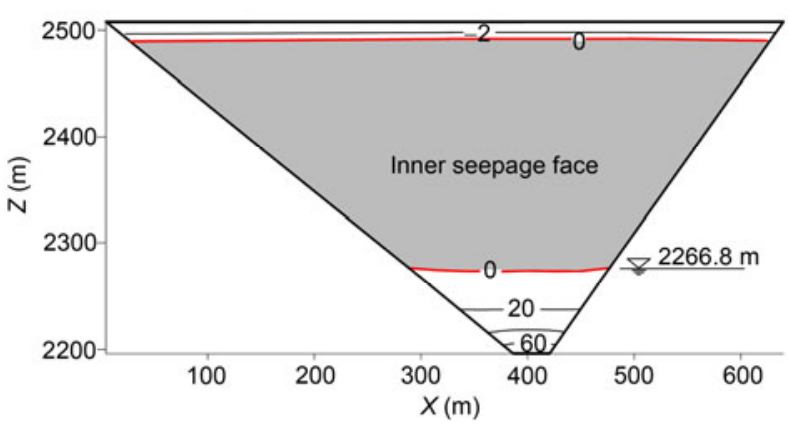

Figure 13 (Color online) Pressure contour in the downstream face of the earth core.

18(10): 1173-1284

6 Gioda G, Gentile C. A nonlinear programming analysis of unconfined steady-state seepage. Int J Numer Anal Meth Geomech, 1987, 11(3): 283-305

7 Cheng Y M, Tsui Y. An efficient method for the free surface seepage flow problem. Comput Geotech, 1993, 15(1): 47-62

8 Wu M X, Zhang X Q. Imaginary element method for numerical analysis of seepage with free surface. J Hydraul Eng, 1994, (8): 67-71

9 Desai C S. Finite element residual schemes for unconfined flow. Int J Numer Anal Meth Geomech, 1976, 10(6): 1415-1418

10 Bathe K J, Khosgoftaar M R. Finite element free surface seepage analysis without mesh iteration. Int J Numer Anal Meth Geomech, 1979, 3(1): 13-22

11 Freeze R A. Influence of the unsaturated flow domain on seepage through earth dams. Water Resour Res, 1971, 7(4): 929-941

12 Cooley R L. Some new procedures for numerical solution of variably saturated flow problems. Water Resour Res, 1983, 19(5): 1271-1285

13 Celia M A, Efthimios T B, Rebecca L Z. A general mass-conservative numerical solution for the unsaturated flow equation. Water Resour Res, 1990, 26(7): 1483-1496

14 Diersch H J G, Perrochet P. On the primary variable switching tech- 
nique for simulating unsaturated-saturated flows. Adv Water Resour, 1999, 23(3): 271-301

$15 \mathrm{Wu}$ M X. A finite-element algorithm for modeling variably saturated flows. J Hydrol, 2010, 394(4): 315-323

16 Lacy S J, Prevost J H. Flow through porous media: a procedure for locating the free surface. Int J Numer Anal Meth Geomech, 1987, 11(6): 585-601

17 Borja R I, Kishnani S S. On the solution of elliptic free boundary problem via Newton's method. Comput Meth Appl Mech Eng, 1991, 88(3): 341-361

18 Bardet J P, Tobita T. A Practical Method for solving free-surface seepage problems. Comput Geotech, 2002, 29: 451-475

19 Herreros M I, Mabssout M, Pastor M. Application of level-set approach to moving interfaces and free surface problems in flow through porous media. Comput Meth Appl Mech Eng, 2006, 195(1-3): $1-25$

20 Ayvaz M T, Karahan H. Modeling three-dimensional free-surface flows using multiple spreadsheets. Comput Geotech, 2007, 34(2): $112-123$
21 Kazemzadeh-Parisi M J, Daneshmand F. Unconfined seepage analysis in earth dams using smoothed fixed grid finite element method. Int J Numer Anal Meth Geomech, 2012, 36(6): 780-797

22 Youngs E G, Rushton K R. Steady state drainage of two-layered soil regions overlying an undulating sloping bed with examples of the drainage of ballast beneath railway tracks. J Hydrol, 2009, (377): 367-376

23 Mao C X. Seepage Computation Analysis and Control. Beijing: China Water Power Press, 2003. 316-317

24 Wang Z M, Jiang H, Yao L, et al. Qualitative experiment of unsaturated water vadose through two-layer porous media. Radiat Prot, 2003, 23(1): 8-13

25 Van Genuchten M Th. A closed-form equation for predicting the hydraulic conductivity of unsaturated soils. Soil Sci Am J, 1980, 44: 892-898

26 Mualem Y. A new model for predicting the conductivity of unsaturated porous media. Water Resources, 1976, 12: 513-522

27 Wu M X, Gao L S. Numerical simulation of saturated-unsaturated transient flow in soils. J Hydraul Eng, 1999, (12): 38-42 